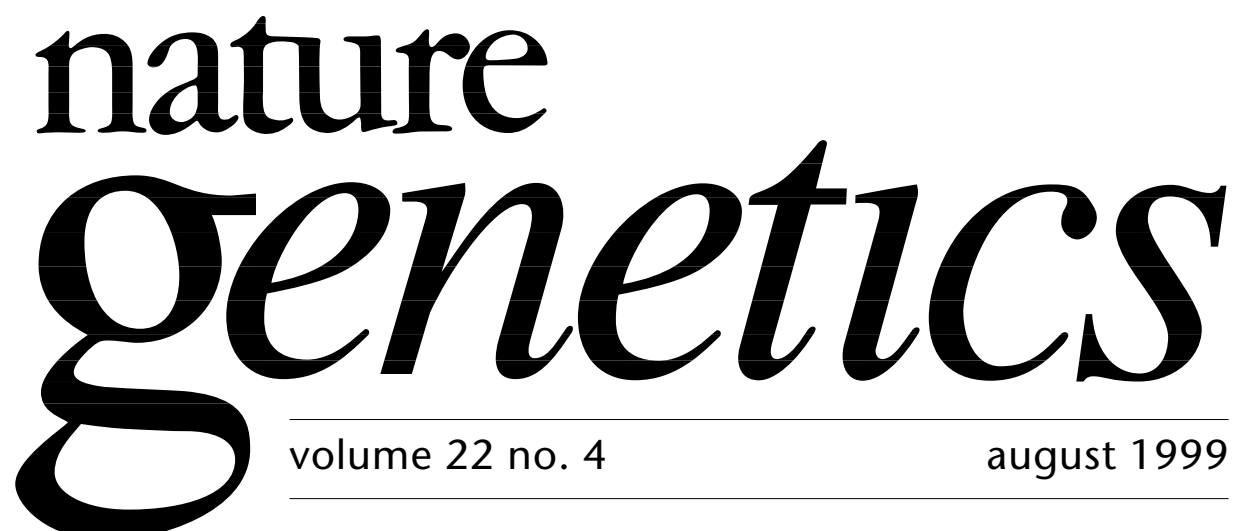

\title{
Gene therapy for rare diseases
}

Gene therapy is a logical way to treat rare genetic diseases; cure a single gene defect by introducing a 'correct' gene. The first gene-therapy trials were conducted using patients with rare monogenetic disorders, but these are now outnumbered by the clinical testing of gene therapeutics for more common conditions, such as cancer, AIDS and cardiovascular disease. This is partly due to a failure to achieve long-term gene expression with early vector systems, a critical requirement for correcting many inborn genetic defects. Now, with the advent of adeno-associated viral (AAV) and lentiviral vectors, which demonstrate persistent gene expression in animal studies, this technological barrier may have been overcome. These vectors are anticipated to form the basis of many gene-therapy protocols for inherited genetic diseases. But will this result in a rapid increase in the number of gene-therapy trials for rare disorders?

The development of gene therapeutics for small patient populations has attracted only limited commercial sponsorship, and scarce resources have hindered the transition from basic science to bedside. Most approaches are currently stalled at the pre-clinical stage, during which data from animal studies, in particular toxicity assessments, are collected prior to application to the Food and Drug Administration (FDA). Data from several hundred clinical trials have led the FDA to conclude that toxicity is largely attributable to the viral vector, rather than the therapeutic transgene. Investigators using similar vectors, therefore, may be replicating unnecessarily the work of others in their evaluations of toxicity. To reduce redundant effort and consequent cost, the FDA and the National Institutes of Health (NIH) have proposed the initiation of 'platform studies' for pre-clinical testing of vector toxicity, with data collectively pooled in a publicly available database. This information would serve as a baseline for assessing a wide range of clinical applications of a given vector. The FDA proposes to modify its requirements so that investigators will need only to conduct toxicity testing for aspects of their protocol that are not addressed by existing data.

The AAV-based vector system is intended to serve as a prototype for platform studies. The proposal has been welcomed by the scientific community, according to Philip Noguchi, of the Center of Biologics Evaluation and Research at the FDA, but, as usual, "the devil is in the details". Issues to be resolved include the funding of the initiative, the standardization of design and quality control of toxicity assays, and establishing a mechanism by which the data can be evaluated, managed and made publicly available. The database, initially a repository for toxicological data, will be expanded to include information on vector biodistribution. The latter, 
although not an absolute prerequisite for phase I trials, is required for the licensing of a therapeutic product (see page 326). Clearly, coordinating efforts in the preclinical development of gene-therapy protocols will hasten the clinical testing of gene therapeutics for rare genetic diseases.

Recent events indicate that a poorly coordinated process of review by different government agencies may lead to additional delays in the initiation of human testing of gene-therapy protocols. The first clinical trial for limb girdle muscular dystrophy, a heterogenous disorder that accounts for the majority of cases of inherited muscle disease, was to begin in July. Treatment of the first patient, however, has been postponed pending public review by the Recombinant DNA Advisory Committee (RAC), an advisory group to the NIH Director that reviews the scientific, legal, social and ethical issues raised by novel approaches to human gene transfer. Although the trial received authorization from the local Institutional Review Board (IRB), the Institutional Biosafety Committee (IBC) and the FDA, it was considered by the RAC to differ sufficiently from standard protocols to warrant public discussion, currently scheduled for September. In principle, clinical trials require only authorization of an Investigational New Drug Application (IND) by the FDA and local IRB and IBC approval, but investigators would be ill-advised to proceed in the face of potential objections from the RAC. While few would dispute that the RAC serves an essential role in the public review of novel human gene transfer, one wonders whether its recommendations would not be better dispensed at an earlier stage of the review process.

The local IBC ensures that a proposed clinical trial adequately addresses biosafety risks. An IND application is not required to have IBC approval in order to be evaluated by the FDA, which responds within 30 calendar days of submission. In contrast, the RAC only considers applications that have received IBC approval. The RAC meets only four times a year and, if a protocol is to be put on the agenda for public discussion - which is the case for approximately $10 \%$ of applications-notice of eight weeks is needed. Delays of several months can ensue if an application is submitted 'out of synch' with the RAC schedule. Moreover, one wonders about the rationale of having one government agency commenting retrospectively on a protocol that has already been authorized by another. It would simplify matters if applications could be submitted to the RAC within a time frame such that public review, if deemed necessary, could take place prior to, or at least simultaneously with, FDA evaluation. Discussions between the FDA and the NIH, in consultation with the RAC, are underway to streamline the process, and revisions are expected. The critical issue will be to establish at what stage during the development of a novel gene therapy protocol RAC input would be optimal.

In the event that a gene therapy approach to treating rare diseases satisfies administrative requirements to proceed with human testing and eventually proves successful in clinical trials, an important issue remains: how to develop commercial products. The pharmaceutical industry is unlikely to see profit potential in supplying a small patient population, especially when only a single injection may be needed over a lifetime to correct the genetic defect. According to James Wilson, of the University of Pennsylvania, the question is "how to price a cure for a rare disease". It is feasible that the government may provide similar incentives to those offered through the FDA Office of Orphan Products to promote the development of conventional drugs for rare diseases. Rather than creating the infrastructure in anticipation of success, however, Wilson believes that "we first have to show that gene therapy works-once we demonstrate success, someone will work out a way to sell it". But for many patients anxiously awaiting the outcome of clinical trials, the resolution of this commercial 'problem' can not come soon enough.

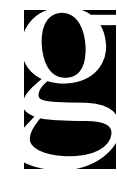

